\section{Internet-Datenbank für Nahrungsmittelallergiker}

W as ist wirklich drin? Die Beantwortung dieser Frage kann für Nahrungsmittelallergiker lebensrettend sein. Zwar hat sich mit Inkrafttreten der neuen Lebensmittelkennzeichnungsverordnung 2005 die Situation deutlich verbessert, dennoch gibt es noch Lücken: So sind manche Inhaltsstoffe nicht anzugeben, wenn sie weniger als $2 \%$ des Nahrungsmittels ausmachen. Auch kann, etwa bei Schokoriegeln, auf der Umverpackung gesetzeskonform die Inhaltsliste fehlen. Vielfach sind schließlich die Angaben aufgrund der kleinen Schrifttype nur schwer zu entziffern. Die Rezepturen der Hersteller werden außerdem verändert, so dass die Inhaltslisten immer wieder überprüft werden müssen

Weiter sind die Nahrungsmittelproduzenten nicht verpflichtet, Inhaltsstofflisten zum Zwecke des Verbraucherschutzes an eine offizielle Stelle zu melden. Abgesehen von einigen Privatinitiativen gab es daher für Nahrungsmittelallergiekranke bisher keine Möglichkeit, diese wichtigen Informationen zentral abzurufen. Hier setzt eine neue Online-Datenbank an: WikiFood. Basierend auf der Idee der Internet-Enzyklopädie Wikipedia soll unter der Adresse www.wikifood.lu eine umfassende Datenbank mit Inhaltsstoffe von Lebensmitteln entstehen: Betroffene, Fach-
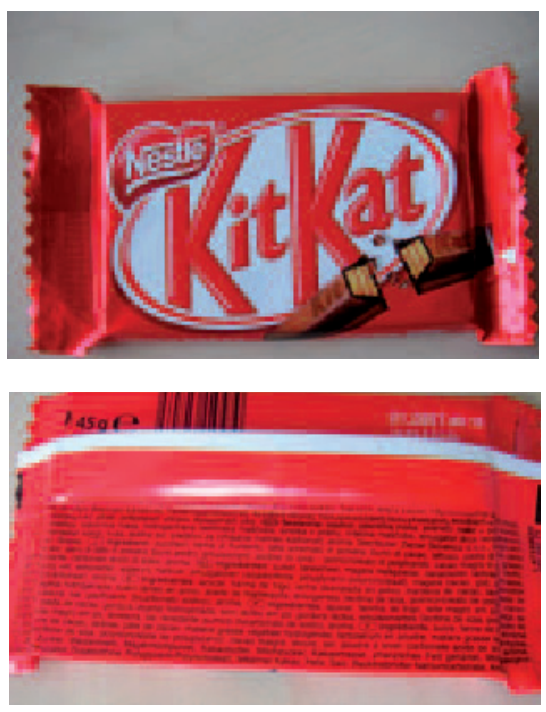

leute und Interessierte, aber auch die Hersteller selbst, sollen darin eine wachsende Sammlung von Nahrungsmitteln und ihren Zutaten aufbauen. Allergikern wird durch die Hervorhebung Allergie auslösender Bestandteile die Auswahl der für sie geeigneten Produkte erleichtert. Zur Identifikation der Produkte werden EAN-13-Barcodes verwendet, die in allen europäischen Ländern zum Einsatz an Kassensystemen dienen.

Initiiert wurde WikiFood von Norbert Rösch vom luxemburgischen Forschungsinstitut Centre de Recherche Public Henri Tudor, das in Kooperation mit dem Centre Hospitalier de Luxembourg innovative Strategien entwickeln will, um Nahrungsmittelallergiker bei der Bewältigung ihrer Erkrankung zu unterstützen: „Bislang fehlte ein unabhängiges und überregionales Portal, dass von Herstellern und Betroffenen zur Verbreitung von Inhaltsstofflisten genutzt werden kann. Die Erfahrungen mit Wikipedia haben gezeigt, dass ein Netzwerk von Freiwilligen durchaus in der Lage ist, eine hohe Datenqualität zu gewährleisten “, erklärte Norbert Rösch. Dank der finanziellen Unterstützung des luxemburgischen Fonds National de la Recherche kann dieses Projekt unabhängig von kommerziellen Interessen verwirklicht werden. red

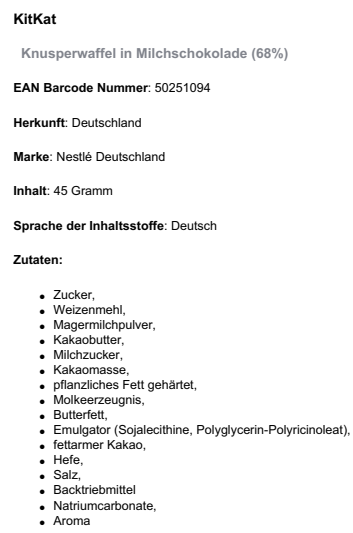

Beispiel für einen Eintrag in Wikifood (www.wikifood.lu): Fotos erleichtern die Identifikation des Produktes und erlauben zudem eine Kontrolle der Einträge.
Bencard-Förderpreis spezifische

Immuntherapie $\mathbf{2 0 0 7}$

Der „Bencard-Förder-

preis spezifische

Immuntherapie",

gestiftet von der

Bencard Allergie

$\mathrm{GmbH}$, München, in

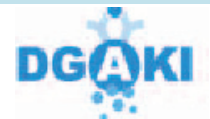

Zusammenarbeit mit

der Deutschen Gesellschaft für Allergo-

logie und klinische Immunologie (DGAKI),

wird jährlich für herausragende Original-

arbeiten verliehen. Der Preis ist aus-

schließlich zur Auszeichnung besonderer

wissenschaftlicher Arbeiten auf dem

Gebiet der Allergologie und klinischen

Immunologie bestimmt und dient der

Förderung jüngerer Wissenschaftler. Zurzeit werden jährlich $\mathbf{5 . 0 0 0 € ~ z u ~ d i e s e m ~}$

Zweck zur Verfügung gestellt.

Der Preis ist für Wissenschaftler aus dem deutschen Sprachraum vorgesehen, die sich noch nicht in Lebensstellung befinden und die das 40 . Lebensjahr zum Zeitpunkt der Einreichung der Arbeit noch nicht vollendet haben. Ausgeschlossen sind Angestellte von Industriefirmen. Für eine Prämierung kommen Arbeiten in Frage, die sich mit diagnostischen und therapeutischen Fragestellungen rund um das Gebiet der spezifischen Immuntherapie befassen.

Die Bewerber reichen bis zum 31. Mai 2007 Originalarbeiten, die bis zu diesem Zeitpunkt publiziert oder zur Publikation angenommen sind, in deutscher oder englischer Sprache ein. Die Arbeiten sollen in fünffacher Ausfertigung vorgelegt werden. Eine Verblindung ist nicht erforderlich. Der Erstautor hat handschriftlich zu versichern, dass er die Arbeit selbstständig erstellt hat. Die Jury kann bei mehr als einer eingereichten preiswürdigen Arbeit den Preis teilen. Je Arbeitsgruppe kann nur eine Arbeit eingereicht werden

Die Verleihung des „Bencard-Förderpreises spezifische Immuntherapie $2007^{\text {“ }}$ findet im Rahmen des Zweiten Gemeinsamen Deutschen Allergiekongresses vom 26. bis 29. September 2007 in Lübeck statt.

\section{Geschäftsstelle der DGAKI}

Frau Erika Ratzinger, Postfach 7004 64, 81304 München

Tel.: (o 89) 54662968

Fax: (o 89) 583824

E-Mail:dgaki@t-online.de 